\title{
Neurofibromatosis type I - some cranial and spinal manifestations
}

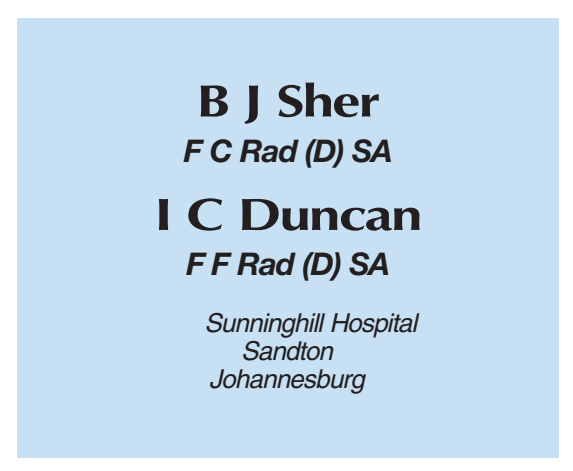

\section{Introduction}

Neurofibromatosis is a hereditary congenital disorder consisting of dysplasias and tumours of tissues arising from the ectodermal and mesodermal layers of the embryo. The most commonly encountered subtype is neurofibromatosis type 1 (NF-1). Shown in this report are several manifestations of NF-1 demonstrated on magnetic resonance imaging (MRI) in 2 individual cases.

\section{Case reports}

\section{Case 1}

An 8-year-old boy with a cervical spine deformity was referred to an orthopaedic surgeon. Axillary freckling and other prominent cutaneous manifestations of NF-I were present. Plain film studies of the cervical spine showed a marked cervical kyphosis with subluxation of C2 on C3. MRI of the cervical spine was requested to rule out a neoplasm prior to cervical fusion.

This scan showed a marked gibbus deformity at $\mathrm{C} 2-\mathrm{C} 3$ due to the subluxation of $\mathrm{C} 2$ on $\mathrm{C} 3$ with narrowing of the subarachnoid space surrounding the cervical spinal cord (Fig. 1a). The cord itself appeared normal. Below the level of the kyphosis mild dural ectasia and posterior vertebral body scalloping were noted (Fig. 1b). MRI of the brain showed numerous hyperintense foci in the basal ganglia and deep white matter, right caudate head, right lentiform nucleus and cerebellum consistent with non-neoplastic hamartomatous lesions (Figs 1c, 1d and 1e). The appearance of these lesions was unchanged when compared with a scan obtained 2 years before.

\section{Case 2}

A 4-year-old boy with external stigmata of neurofibromatosis presented with a mild right-sided proptosis and suspected orbital tumour. Cranial MRI showed dysplasia of the right sphenoid wing, with an osseous defect and herniation of the temporal lobe into the posterior aspect of the orbit (Fig. 2a). The subarachnoid space around the temporal lobe itself appeared widened, with associated dilatation of the temporal horn of the right lateral ventricle. There was an enhancing mass within the superolateral aspect of the right orbit, in keep-

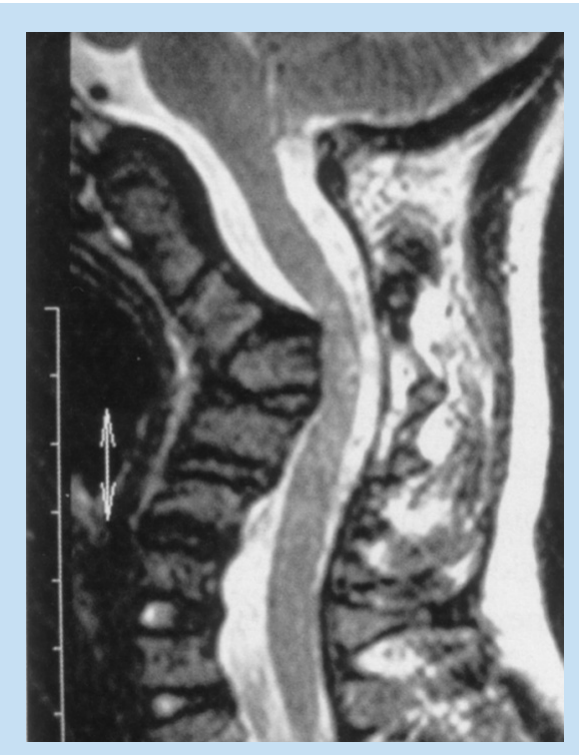

Fig 1a. Sagittal T2-weighted MR images showing the focal gibbus at the C2, C3 and C4 levels. The canal is narrowed at this level but no cord hyperintensity is seen.

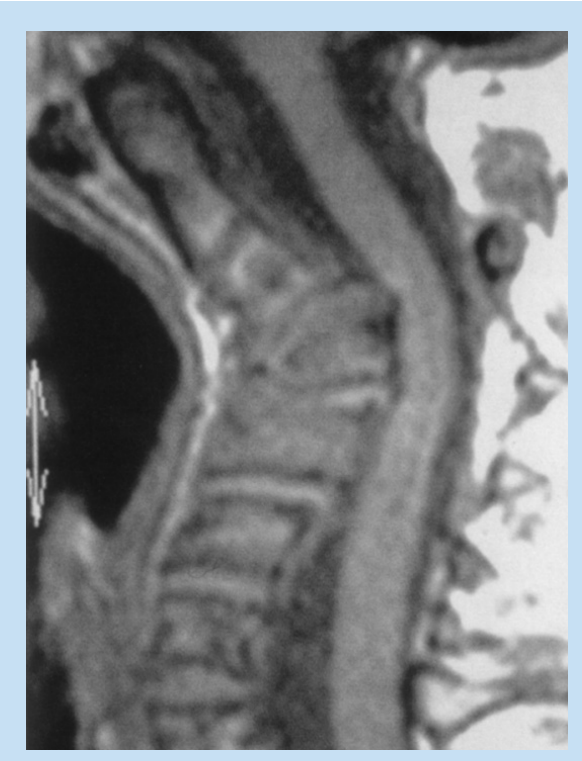

Fig 1b. Sagittal T1-weighted image showing slight posterior vertebral body scalloping and dural ectasia at the C5 and C6 levels.

ing with a plexiform neurofibroma (Figs $2 \mathrm{~b}$ and 2c). This extended posteriorly along the ophthalmic division of the trigeminal nerve. The optic nerve itself appeared displaced and stretched but otherwise normal. The ocular globe was normal. The brain scan showed further dysplasia of the right half of the calvarium with hem- 


\section{CASE REPORT}

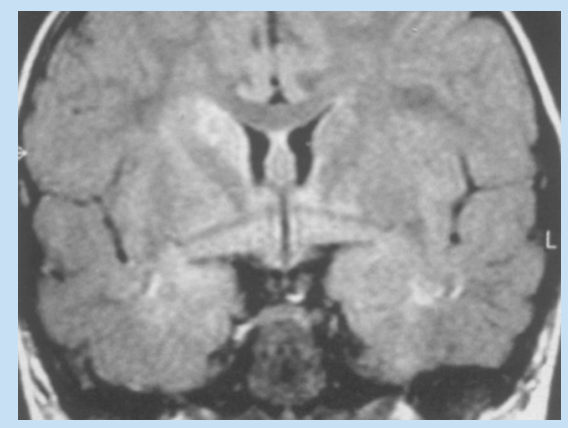

Fig 1c. Coronal FLAIR image showing focal hyperintensities in the head of the right caudate nucleus and subcortical white matter of the temporal lobes.

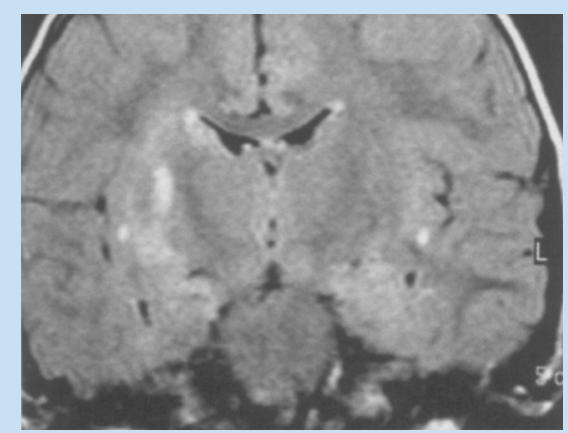

Fig 1d. Coronal FLAIR image at a more posterior level showing a focal hyperintensity in the right lentiform nucleus and subinsular white matter.

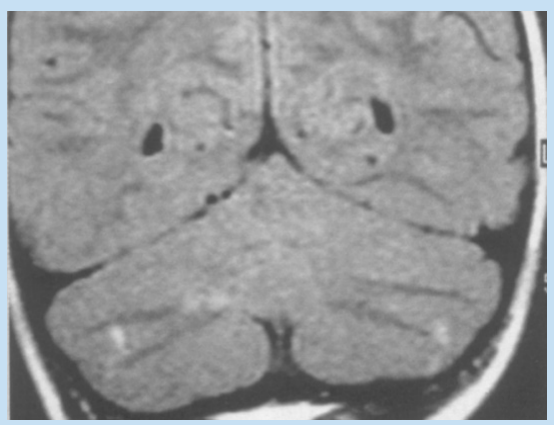

Fig 1e. Coronal FLAIR image showing hyperintensities within the cerebellar white matter. All lesions had remained radiologically stable for 2 years.

icranial enlargement, and enlarged surface sulci and gyri of the right cerebal hemisphere together with unilateral enlargement of the right lateral ventricle secondary to the increase in volume of the right hemicranial cavity (Figs $2 \mathrm{~d}$ and 2e).

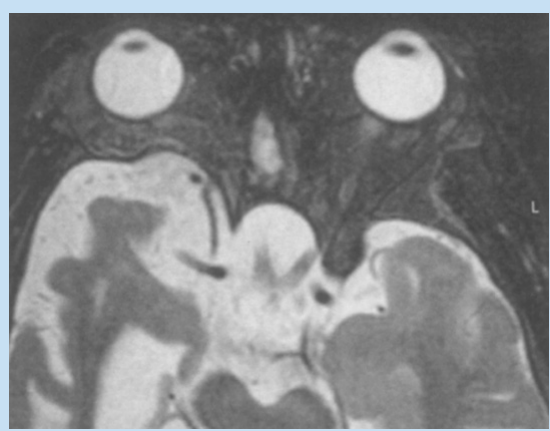

Fig 2a. Axial T2-weighted image shows dysplasia of the right sphenoid wing with herniation of the peritemporal subarachnoid space into the posterior right orbit.

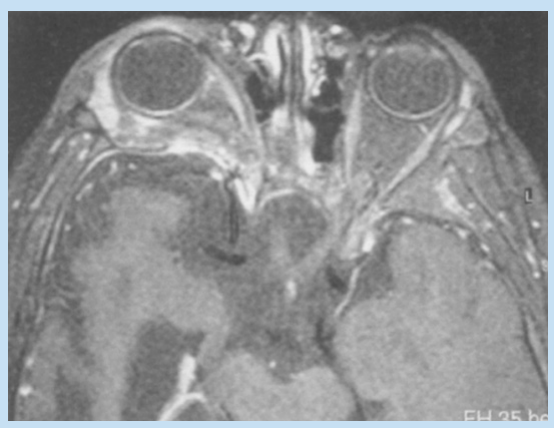

Fig 2b. Axial gadolinium-enhancement T1-weighted image at the same level showing an enhancing plexiform neurofibroma within the lateral aspect of the orbit (arrow).

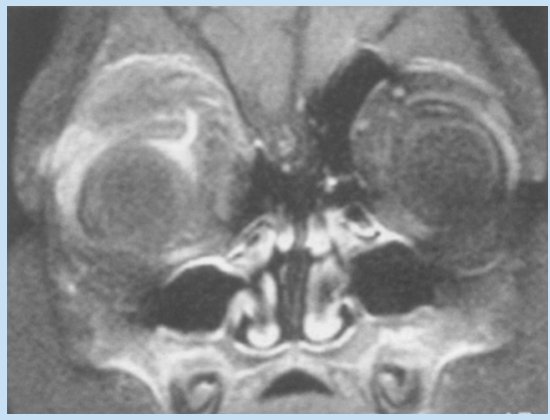

Fig 2c. Coronal gadolinium-enhanced T1-weighted image at the same level showing the enhancing plexiform neurofibroma in the lateral aspect of the orbit (arrow)

\section{Discussion}

The phakomatoses (phakos (Greek) $=$ birthmark, spot or freckle) are a group of congenital disorders characterised by the development of dysplasias and neoplasias of organs arising from the embryonic ectoder-

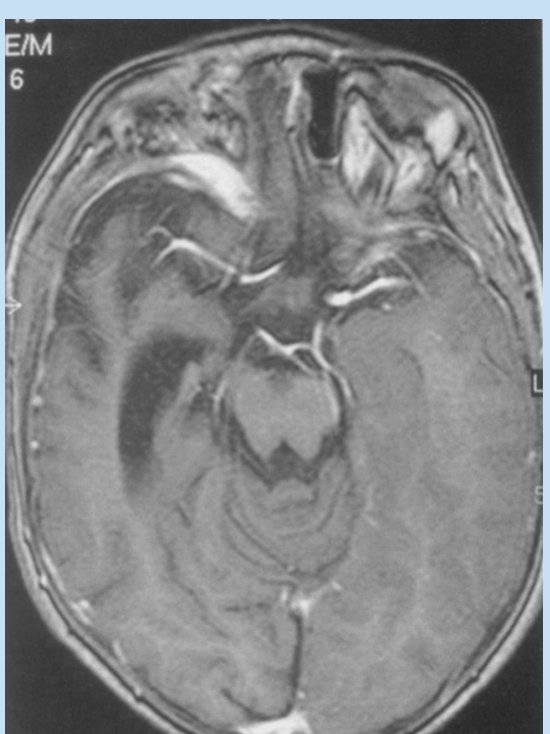

Fig 2d. Axial gadolinium-enhanced T1-weighted image showing dilation of the peritemporal subarachnoid space and temporal horn of the right lateral ventricle.

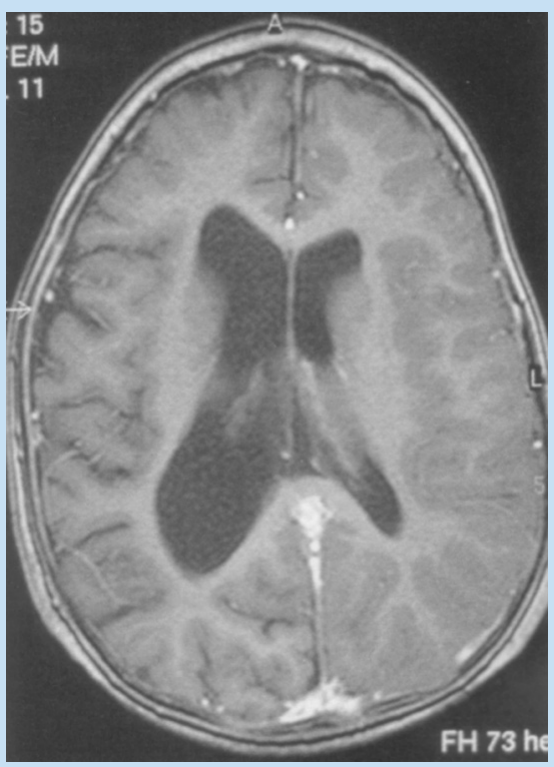

Fig 2e. Axial gadolinium enhanced T1-weighted image at a higher level showing dysplasia of the right hemicalvarium with associated enlargement of the surface sulci and right lateral ventricle secondary to the increased hemicalvarial volume.

mal plate (involving the skin, central and peripheral nervous systems and eyes), and to a lesser degree structures derived form the mesodermal layer (including bone, cartilage and blood vessels) and the endodermal layer 
(including the gastro-intestinal tract). Owing to the predominantly ectodermal involvement these syndromes are also termed the 'neurocutaneous syndromes' or 'congenital neuroectodermal dysplasias." The commonest of the neuroectodermal syndromes is NF-I, also known as Von Recklinghausen's neurofibromatosis. With a prevalence of 1:3000 - 1:5000 individuals, NF-1 is over 10 times commoner than neurofibromatosis type II (NF-2). NF-1 is genetically distinct from the less common NF-2 in that NF-1 is related to an abnormal gene occurring on the long arm of chromosome 17 whereas NF-2 results from an abnormal gene on chromosome 22. Both types are inherited as autosomal dominant disorders, with NF-1 having a penetrance of almost $100 \%$ and spontaneous mutation rate of about $50 \% .{ }^{2}$ The NF-1 gene is a tumour suppressor gene that becomes inactivated as a result of the mutation. ${ }^{3}$ A similar mechanism affecting tumour suppression genes on chromosome 22 results in the clinical manifestations of NF-2. For further discussion about the genetics and manifestations of NF-2 readers are referred to an earlier article in this journal by Kritzinger. ${ }^{4}$ The diagnostic criteria for NF-1 are listed in Table I.
Central nervous system lesions occur in about $15-20 \%$ of patients with NF-1., ${ }^{1,3}$ These include optic gliomas, occuring in $5-15 \%$ of patients, ${ }^{1}$ of which half become symptomatic and which can involve the optic nerves and/or pathways. Brain lesions include gliomas, particularly the juvenile pilocytic astrocytoma and brainstem gliomas. Gliomas in NF-1 can also occur in the optic pathways, hypothalamus, third ventricle, basal ganglia, tectum, cerebral peduncles, cerebellum and cerebral hemispheres. ${ }^{1,3,5}$ These gliomas are generally low-grade tumours. Non-neoplastic hamartomatous lesions, also termed 'unidentified NF-1 objects' or 'UNOs' can be found in up to $80 \%$ of patients with NF-1. 1.5 These lesions are found anywhere within the brain parenchyma but particularly in the basal ganglia, thalamus, internal capsule, brainstem, cerebellum and supratentorial white matter. ${ }^{1,3}$ These lesions are iso-intense on $\mathrm{T} 1$-weighted and hyperintense on $\mathrm{T} 2$-weighted and FLAIR MR images and show no mass effect, vasogenic oedema or contrast enhancement. They are seen in about $75 \%$ of children with NF-1, first appearing at around 3 years of age and resolving by the age of 20 years. $^{6}$ Progression or change in any of these

\section{Table I. Diagnosis for neurofibromatosis 1}

\section{Criteria for NF-1 (two or more of the following)}

- Six or more café au lait macules (diameter $>5 \mathrm{~mm}$ in prepubertal patients and $>15 \mathrm{~mm}$ in postpubertal patients)

- Two or more neurofibromas or one plexiform neurofibroma

- Axillary or inguinal freckling

- Optic glioma

- Two or more Lisch nodules (iris hamartomas)

- A characteristic osseous lesion (sphenoid) dysplasia or cortical thinning of long bone, with or without pseudarthrosis

- First-degree relative (parent, sibling, or child) with NF-1 lesions in a child older than $10-12$ years of age is suspicious of a neoplasm and therefore demands closer follow-up. It is currently thought that these lesions represent regions of myelin vacuolisation, with areas of separation of myelin as it spirals around the nerve axons. ${ }^{7}$ It is also thought that the myelin eventually repairs itself, causing the lesions to show spontaneous regression. ${ }^{3}$ Intracranial dural lesions include dural ectasia, meningoceles and meningioangiomatosis. ${ }^{3}$

In addition to the intracranial neuro-ectodermal dysplasias and tumours found in NF-1, various dysplasias of mesodermally derived cranial structures can be found, including vascular and osseous orbital dysplasias. Associated vascular lesions include stenoses of the extracranial and intracranial arteries and the development of cerebral aneurysms or arteriovenous malformations. Of the osseous dysplasias, only sphenoid wing dysplasia is of any critical clinical importance. ${ }^{3}$ A defect in the sphenoid wing allows the temporal lobe to herniate into the posterior aspect of the orbit. Arterial pulsations within the temporal lobe are transmitted into the orbit causing pulsatile exophthalmos, sometimes with secondary dysplasia or atrophy of the globe and other orbital contents. ${ }^{1,3}$ Sphenoid wing dysplasia is often associated with plexiform neurofibromas in the orbit or peri-orbital regions. ${ }^{3}$

The characteristic peripheral and craniofacial nerve lesions of NF-1 are the neurofibromas and plexiform neurofibromas, the latter often occuring within the orbit and extending backwards into the cavernous sinus through the superior orbital fissure, but generally not beyond Meckel's 
cave. ${ }^{5}$ These generally follow the course of the ophthalmic division of the trigeminal nerve. ${ }^{3}$ On MR imaging plexiform neurofibromas tend to be slightly hyperintense relative to muscle and hypo-intense to brain on T1-weighted images and of high signal intensity relative to brain and muscle on T2-weighted images, sometimes demonstrating a central hypointense region in T2-weighted images due to a central core of fibrous tissue. Contrast enhancement is variable. $^{1,3}$

Spinal manifestations are common in NF-1. Scoliosis and kyphosis are the most commonly encountered skeletal abnormalities that result from dysplasia of the vertebral bodies. ${ }^{1,3}$ Other bony vertebral abnormalities include increased interpedicular distances, hypoplasia of the pedicles and other posterior elements, posterior scalloping of the vertebral bodies and enlargement of neural outlet foramens. These changes can either be due to primary bone dysplasia with dural ectasia or secondary to the effects of neurofibromas. The neurofibromas generally affect the dorsal nerve roots and are intradural extramedullary tumours which can extend extradurally thorugh the neural foramens, then being known as 'dumb-bell' or 'hourglass' tumours. Malignant neurofibromas are uncommon, with an estimated frequency of malignancy of up to $10 \% .^{3}$ Meningeal dysplasia is probably responsible for the development of lateral arachnoid cysts or meningoceles that can herniate through the neural outlet foramens, often widening the foramens in a manner similar to emerging neurofibromas. Meningoceles can also result in posterior vertebral scalloping. The CSF-filled meningocele can be differentiated from neurofibromas as the latter show some central hypointensity on T2-weighted images and are of higher signal intensity than CSF on T1-weighted images. ${ }^{1,3}$

\section{References}

1. Braffman B, Naidich TP. The phakomatoses: Part 1. Neurofibromatosis and tuberous sclerosis. Neuroimaging Clin North Am 1994; 4: 299324.

2. Aoki S, Barkovich AJ, Nishimura K, et al. Neurofibromatosis types 1 and 2: Cranial MR findings. Radiology 1989; 172: 527- 534.

3. Barkovich AJ. The phakomatoses. In: Barkovich AJ, ed. Pediatric Neuroimaging. 3rd ed. Philadelphia, Lippincott Williams \& Wilkins, 2000: 383-441.

4. Kritzinger HG. Neurofibromatosis type $2-\mathrm{a}$ case study SA Journal of Radiology 2002; 6: 3740.

5. Osborn AG. Disorders of histiogenesis: Neurocutaneous syndromes. In: Osborn AG, ed. Diagnostic Neuroradiology. St Louis: Mosby, 1994: 72-113.

6. Sevick R, Barkovich A, Edwards M, Koch T, Berg B, Lempert T. Evolution of white matter lesions in neurofibromatosis type 1: MR findings. Am J Roentgenol 1992; 159: 171-175.

7. DiPaolo DP, Zimmerman RA, Rorke LB, Zackai EH, Bilaniuk LT, Yachnis AT. Neurofibromatosis type 1: Pathological substrate of high-signal intensity foci in the brain. Radiology 1995; 195: 721-724. 